\title{
Vertical and Horizontal Hop Performance: Contributions of the Hip, Knee, and Ankle
}

\author{
Argyro Kotsifaki, PT, MSc, ${ }^{\dagger}{ }^{\dagger}$ Vasileios Korakakis, PT, PhD, ${ }^{\dagger}$ Philip Graham-Smith, PhD, ${ }^{\ddagger}$ \\ Vasileios Sideris, $\mathrm{MSc}^{\dagger}$ and Rod Whiteley, $\mathrm{PT}, \mathrm{PhD}^{\dagger}$
}

Background: Single-leg vertical and horizontal hop tests are commonly used to assess performance of healthy athletes and as a measure of progress during rehabilitation from knee injury. It is unclear if they measure similar aspects of leg function, as the relative joint contributions of the hip, knee, and ankle joints during propulsion and landing are unknown.

Hypothesis: The proportion of work done by the hip, knee, and ankle will not be the same for these 2 jump types and will vary for propulsive and landing phases.

Study Design: Cross-sectional cohort study.

Level of Evidence: Level 3.

Methods: Twenty physically active participants completed instrumented single-leg hop analysis in both vertical and horizontal directions. Joint peak power, work generated or absorbed, and percentage contribution of each joint during propulsive and landing phases were compared between tasks using paired $t$ tests.

Results: Vertical hop was performed with roughly similar contributions of the hip, knee, and ankle for both propulsion (31\%, 34\%, 35\%, respectively) and landing (29\%, 34\%, 37\%, respectively). Horizontal hop distance was mostly (87\%) determined by the hip and ankle ( $44 \%$ and $43 \%$ ), but landing was mostly (65\%) performed by the knee with lesser contribution from the hip and ankle (24\% and $11 \%)$. Propulsive phase showed a proximal-to-distal temporal sequence for both hop types, but landing was more complex.

Conclusion: Performance during vertical and horizontal hops (jump height and jump distance, respectively) measures different aspects of hip, knee, and ankle function during the propulsive and landing phases.

Clinical Relevance: Assessment of knee joint function during rehabilitation should not be done using a horizontal hop. The knee contributes about a third to vertical hop height, but only about an eighth to horizontal hop distance. Practitioners carrying out performance testing using either vertical or horizontal hops should be mindful of the relative contributions for meaningful training inferences to be derived.

Keywords: vertical hop; horizontal hop; power; joint work

$\mid$ op tests were traditionally designed to assess functional performance in an injured leg. ${ }^{1,23}$ These tests contrast with so-called "analytic" tests such as isokinetic dynamometry, which typically examine the force output for single joints alone. ${ }^{35}$ The claimed advantages of these analytic or "isolated" tests are their ability to more closely describe the function of the individual components of the kinetic chain; however, their weakness lies in their inability to correlate fully with performance tasks required of athletes during their sport. ${ }^{2}$ Clinicians may prefer the relative simplicity of functional tests such as single-leg hop for distance or height over dynamometry, which requires expensive instrumentation. Additionally, dynamometry may be argued as less "functional," especially since the correlation between, for example, knee strength and hop height or hop distance is unclear. In addition, isokinetic dynamometry tests muscle force generation parameters in a nonweightbearing position in contrast to hop tests. To make clinical inferences, we must understand the relative contributions of the different components of the kinetic chain to performance. It has been argued that the horizontal hop is

From ${ }^{\dagger}$ Department of Rehabilitation, Aspetar Orthopaedic and Sports Medicine Hospital, Doha, Qatar, and ${ }^{\ddagger}$ Aspire Academy, Doha, Qatar

*Address correspondence to Argyro Kotsifaki, PT, MSc, Department of Rehabilitation, Aspetar Orthopaedic and Sports Medicine Hospital, Sports City Street, Doha, 20222, Qatar (email: argyro.kotsifaki@aspetar.com) (Twitter: @RoulaKotsifaki).

The authors report no potential conflicts of interest in the development and publication of this article.

DOI: 10.1177/1941738120976363

(C) 2021 The Author(s) 
Table 1. Participant characteristics $(\mathrm{N}=20)$

\begin{tabular}{|l|c|c|}
\hline & Mean \pm SD & Range \\
\hline Age, $\mathrm{y}$ & $28.9 \pm 3.6$ & $19.5-34.3$ \\
\hline Body mass, $\mathrm{kg}$ & $76.1 \pm 7.4$ & $55.5-90.4$ \\
\hline Height, $\mathrm{cm}$ & $178 \pm 6$ & $167-192$ \\
\hline Body mass index, $\mathrm{kg} / \mathrm{m}^{2}$ & $24.1 \pm 1.7$ & $19.9-26.9$ \\
\hline Tegner activity scale & $7.4 \pm 1.0$ & $6-9$ \\
\hline
\end{tabular}

simply an inclined vertical hop and can therefore be used interchangeably as a performance metric. ${ }^{16,28}$ Similarly, there exist little data, mostly presented for bilateral tasks ${ }^{11,14,37}$ to inform the relative contributions of the different joints in the propulsive and landing phases of these tasks. In a clinical setting, 2-leg performance tasks allow the possibility of offloading the injured leg and masking any deficits. As such, when prescribing these tasks for testing or even training purposes, the clinician is not able to accurately ascertain the relative contribution of the hip, knee, and ankle.

These hop tests are used widely in a clinical setting because of their ease of administration and the use of the uninjured leg as a reference for comparison. ${ }^{12}$ Single-leg landing tasks are more representative of biomechanics during multidirectional sport-specific activities because of the significantly greater forces generated, especially the eccentric demands of the posterolateral hip musculature during the deceleration phase. ${ }^{33}$ Horizontal hop and vertical hop can be classified similarly as having a propulsive and a landing phase. Ostensibly, the main difference lies in the direction of the hop. Practitioners need to understand the relative contribution of individual joints to performance to allow clinical reasoning and decisions regarding strength and coordination.

Accordingly, we sought to measure the individual contributions of these 3 joints during single-leg hop for distance (horizontal hop) and height (vertical hop) in a group of healthy athletic adult males. Specifically, the purpose of this study was to identify, compare, and interpret specific lower limb biomechanical differences or similarities between a vertical and horizontal hop during the propulsive and landing phase to inform clinicians about which task may be more appropriate for assessing or training specific functional characteristics.

\section{METHODS}

\section{Study Design}

A cross-sectional design was used with the aim to evaluate lower limb biomechanical differences between vertical and horizontal hop tests. This single-session laboratory-based study was conducted between January and March 2019, at Aspetar Orthopaedic Sports Hospital. Ethical approval was obtained from the local ethics committee (F2017000227 Anti-Doping Lab Qatar) and all participants gave written informed consent. Reporting of the present study followed the STROBE (Strengthening the Reporting of Observational Studies in Epidemiology) statement for cross-sectional studies. ${ }^{39}$

\section{Participants and Eligibility Criteria}

A convenience sample of 20 male adults was recruited to participate in the study. All participants (Table 1) were healthy physically active individuals, participated in sports at least 3 times per week, had not suffered a back or lower extremity injury within the previous 6 months, and had no history of surgery to either lower extremity.

\section{Procedures}

Equipment, Participants' Preparation, and Markers Set

All participants were evaluated at the same location by the same examiner (physical therapist with more than 15 years of experience) and wore athletic shorts and standard shoes. Data pertaining to age, gender, height, and body mass were recorded and body mass index was calculated $\left(\mathrm{kg} / \mathrm{m}^{2}\right)$. Limb dominance was determined by asking the participants with which limb they would prefer to kick a ball. ${ }^{36}$

In total, 42 reflective markers were placed according to a full-body Plug-in-Gait marker-set, extended with additional anatomical markers on the sacrum and the medial malleoli and 3 marker clusters laterally on each thigh and shank. ${ }^{8}$ The motion data acquired from the participant were static anatomical poses and dynamic trials. During all trials, marker motion was captured with a 14-camera motion capture system (250 Hz; Vicon). During the dynamic trials, ground reaction forces (GRFs) were collected synchronously with marker trajectories using 5 in-ground force plates (1000 Hz; Kistler).

\section{Testing Protocol}

All participants initially performed a 7-minute warm-up session, including running, side running, deep squats, and double leg jumps. A physical therapist gave a verbal description and demonstrated both testing procedures/tasks. Subsequently, participants could practice until they felt comfortable with the 
tasks, while verbal feedback was provided. Vertical and horizontal hops were evaluated in a random order in terms of side testing to avoid potential bias.

For both tests, participants stood on a force plate, placed their hands over their hips, and followed a standardized procedure. For the vertical hop they started from an upright single-leg standing position before countermoving to a self-selected depth as fast as possible, then jumped vertically with maximum effort and landed on the same leg. For the horizontal hop, they were instructed to stand on 1 leg and jump horizontally as far as possible, landing on the same leg on another force plate. A successful trial required participants to land inside the borders of the force plate and to hold the final landing for at least 2 seconds. Data were collected for both legs, and 4 successful trials were used for analysis.

\section{Data Processing}

A standard inverse dynamics analysis was used to calculate the internal moments at the hip, knee, and ankle joints. ${ }^{41}$ Kinematic and kinetic variables were calculated for hip, knee, and ankle joints for both limbs and tasks. The variables of interest were joint power, joint work, and percentage work contribution of each joint.

Data were processed in Visual 3D (C-Motion, Inc). Marker trajectories and GRFs were low-pass filtered using a zero-lag, fourth order, Butterworth filter with the same cutoff frequency of $15 \mathrm{~Hz}$, a procedure consistent with published recommendations. ${ }^{3}$

All data were extracted at 2 phases. Propulsive phase was defined as 0.4 seconds prior to takeoff until takeoff, and landing phase from initial contact to peak knee flexion. Toe-off and initial contact were expressed as the point when vertical GRF became less than $20 \mathrm{~N}$ and more than $20 \mathrm{~N}$, respectively.

Power was calculated as a scalar product of joint angular velocity and net joint moment. Work generation was calculated as net joint power integrated over time, during the propulsive phase, in regions with positive internal power and work absorption during the landing phase in regions with negative internal power. The contribution of each joint was determined as percentage of the sum of all 3 lower limb joints during each phase. ${ }^{30}$ Power and work were normalized by body mass. Ensemble average curves were produced for power outcomes, and individual work percentage calculations were made. Preliminary between-limb statistical testing in all parameters of interest indicated no significant differences; therefore, dominant and nondominant limbs were combined in the analysis.

\section{Statistical Analysis}

Descriptive statistics were used to summarize the characteristics of the participants and the measurements. Data were checked for normality using Shapiro-Wilk tests, analyzed using SPSS (Version 23.0), and statistical significance was set at $P<0.05$. The mean of all 4 trials for each participant, for each task, was used to test task differences.

Data were analyzed by using paired-samples $t$ tests, and Cohen $d$ effect sizes (ESs) were calculated. ${ }^{6}$ The ES values of
$0.2,0.5$, and 0.8 were identified as the lower thresholds for small, moderate, and large effects, respectively. Pattern analyses were performed using spm1d (parametric hypothesis testing) (V 0.3; www.spm1d.org). Data processing and statistical parametric mapping were performed with MATLAB (R2018a; Mathworks Inc).

\section{RESULTS}

Significant differences in joint power, work, percentage contribution, and temporal sequences of each joint were noted between the 2 hopping tasks for both propulsion and landing (Figures 1-3 and Table 2).

\section{Peak Power and Work Contribution to Tasks \\ Within-Task Differences in Propulsion}

In the horizontal hop, peak ankle power was significantly greater than both hip $(d=3.23)$ and knee $(d=5.38)$ peak power, and hip peak power was significantly greater than knee $(d=3.15)$ peak power (all $P<0.001)$. Work generation from the hip and ankle joint did not differ $(P=0.436)$ but was significantly greater than knee work $(d=4.43$ and $d=4.40$, respectively; $P<0.001)$.

In the vertical hop, peak ankle power was significantly greater than both hip $(d=1.54 ; P<0.001)$ and knee $(d=1.38$;

$P<0.001)$ peak power; however, no significant differences were evident between hip and knee peak power $(P=0.111)$. Work generation from the knee joint did not differ significantly from both hip $(P=0.128)$ and ankle $(P=0.764)$ work contribution. Ankle joint work contribution was marginally greater than that of the hip joint $(d=0.42 ; P<0.05)$.

\section{Within-Task Differences in Landing}

In the horizontal hop, peak knee power absorption was significantly greater than both hip and ankle peak power absorption ( $d=1.49$ and $d=3.18$, respectively; $P<0.001)$, and hip peak power absorption was significantly greater than ankle peak power $(d=1.58 ; P<0.001)$. The work contribution of the knee joint in landing was significantly greater than both the hip and the ankle $(d=3.59$ and $d=5.22$, respectively; $P<0.001)$ joint contribution, and the hip joint work contribution was significantly greater than the ankle $(d=1.99 ; P<0.001)$ joint work contribution.

In the vertical hop, peak power absorption did not differ between the ankle and hip $(P=0.747)$; however, peak knee power absorption was less than both the hip $(d=0.60$; $P<0.05)$ and ankle $(d=0.90 ; P=0.001)$. The work contribution from the ankle joint did not differ from either the hip $(P=0.193)$ or the knee $(P=0.925)$ work contribution. Knee joint work contribution was marginally greater than that of the hip joint $(d=0.34 ; P<0.05)$.

\section{Between-Task Differences in Propulsion}

The total work in propulsion was significantly greater in horizontal compared with vertical hop $(d=0.53 ; P=0.007)$. 

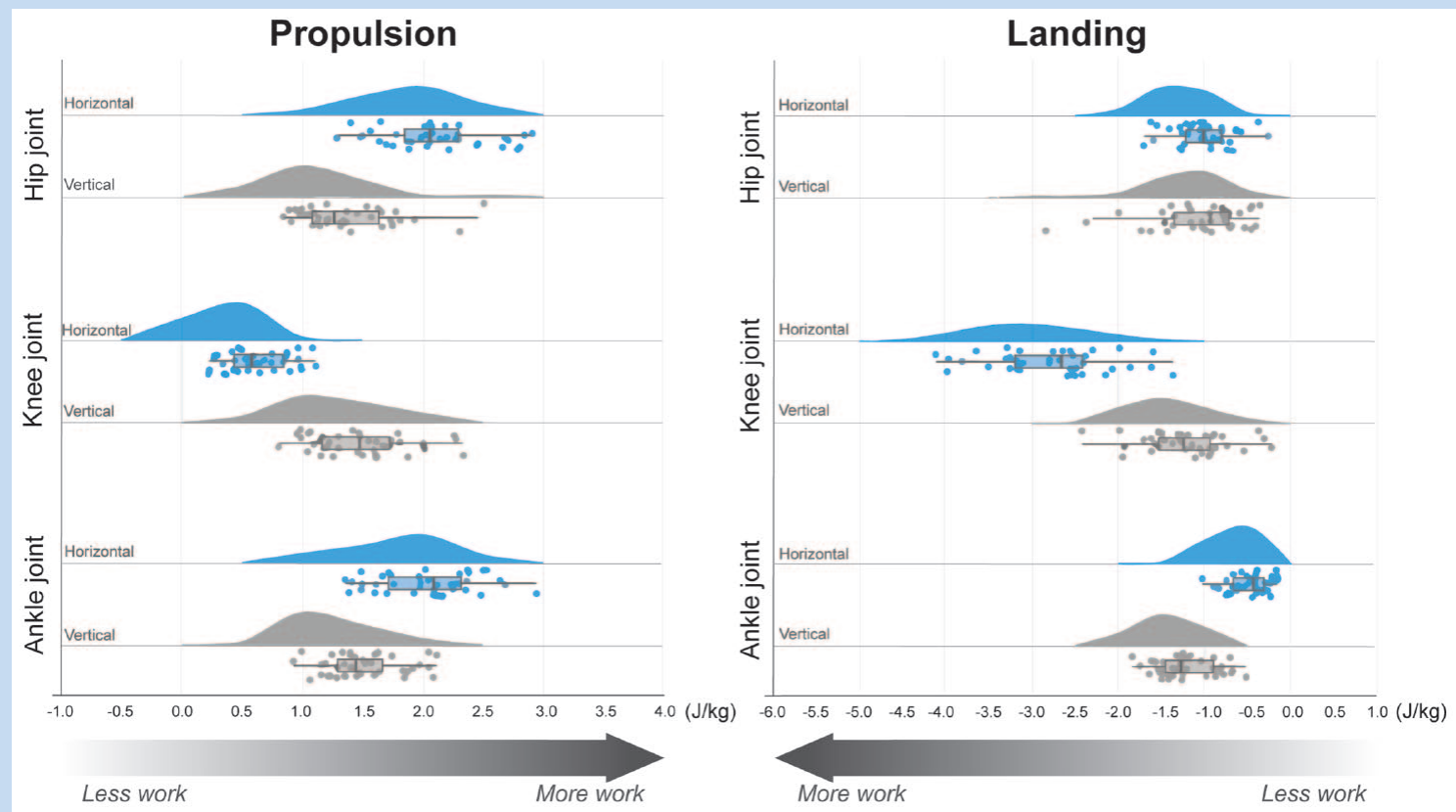

Figure 1. Average work done $(\mathrm{J} / \mathrm{kg}$ ) for the individual participants (filled dots) along with the associated boxplot and probability distribution of the work done for the propulsive and landing phases of each of the 3 joints, for both tasks. For the propulsion phase, increasing work is positive, whereas for the landing phase increasing work (absorption) is negative. Note especially the greatest absorption work being done by the knee during landing from the horizontal hop, whereas during propulsion the knee is the lowest contributor. In contrast, for the vertical hop, the contributions of the 3 joints are roughly similar (at about 1/3 of the total work) during both propulsion and landing.

Peak hip and ankle power were significantly increased $(d=1.16$ and $d=2.27$, respectively; $P<0.001)$ in horizontal compared with vertical hop. Conversely, peak knee power generation was significantly less in horizontal compared with vertical hop with a very large ES $(d=2.12 ; P<0.001)$.

The relative work contribution of hip and ankle joint in propulsion was significantly higher $(d=1.85$ and $d=1.52$, respectively; $P<0.001$ ), while the work contribution of the knee joint was significantly lower $(d=2.69 ; P<0.001)$ in horizontal compared with vertical hop (Table 2, Figure 3).

\section{Between-Task Differences in Landing}

The total work in landing was significantly greater in the horizontal compared with vertical hop $(d=0.97 ; P<0.001)$. Peak hip power absorption was moderately higher in horizontal compared with vertical hop $(d=0.46 ; P=0.027)$, while knee peak power was substantially higher in horizontal compared with vertical hop $(d=3.48 ; P<0.001)$. Peak ankle power absorption was higher in vertical compared with horizontal hop with a large ES $(d=1.05 ; P<0.001)$.

The ankle joint work contribution in landing was greater in the vertical compared with horizontal hop $(d=2.69 ; P<0.001)$. The knee joint had a larger and significantly greater work contribution in horizontal compared with vertical hop $(d=2.76 ; P<0.001)$ (Table 2, Figure 3).

\section{DISCUSSION}

The results of the present study indicate that the vertical and horizontal hop do not measure the same constructs of lower limb function. This contention is also supported by previous research, ${ }^{22,32}$ which showed a relatively low correlation (0.630.71 ) between vertical and horizontal hop, suggesting that these 2 tests measure different constructs of function.

To our knowledge, there is limited evidence explaining quantitatively the relative contributions of kinetic variables to these single-leg functional tests. There is some research examining contributions of the different joints during bilateral tasks. ${ }^{11,14,29,37}$ However, comparing the current work with these findings shows large differences in relative joint contributions.

We suggest that bilateral and single-leg tasks are not comparable and likely involve different motor strategies.

\section{Knee Strength and Jump Performance}

Despite both vertical and horizontal hop tests displaying high test-retest reliability $(0.88-0.99),{ }^{4,5,7,10,27,38}$ the validity of the tests remains inconclusive. Statistically significant correlations have been documented between quadriceps strength and horizontal hop performance ${ }^{10,15,21,26,40}$; however, closer scrutiny of the underlying data may change our clinical interpretation of these findings. Specifically, a correlation of $r=0.36$ (isokinetic 

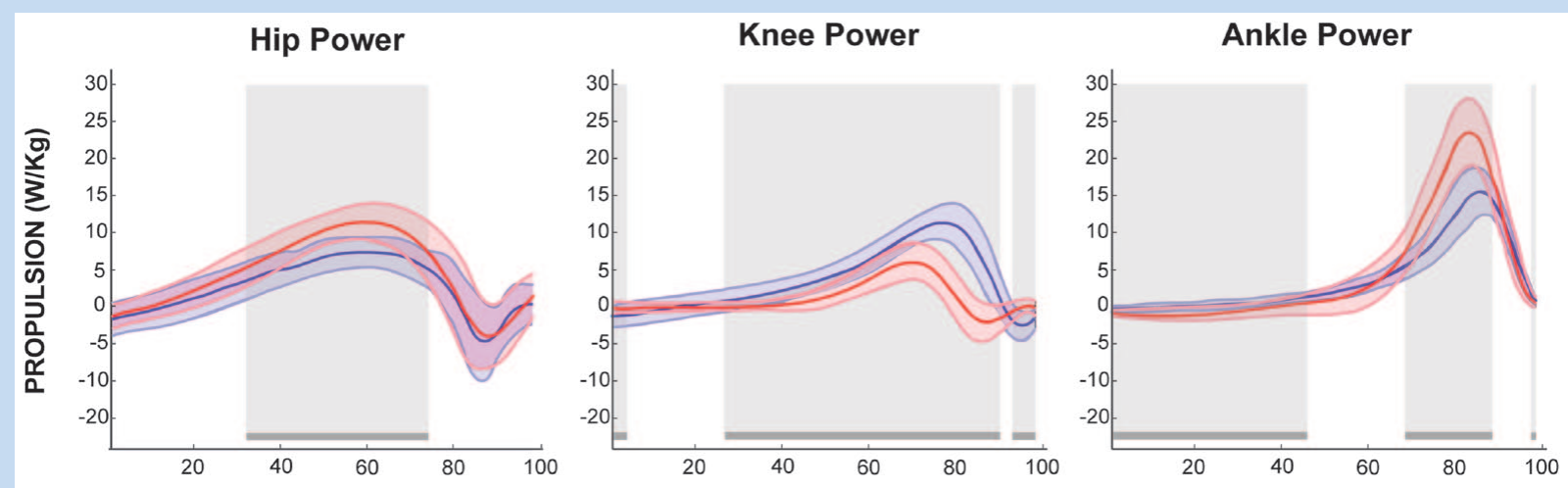

Percentage $(\%)$ of propulsive phase
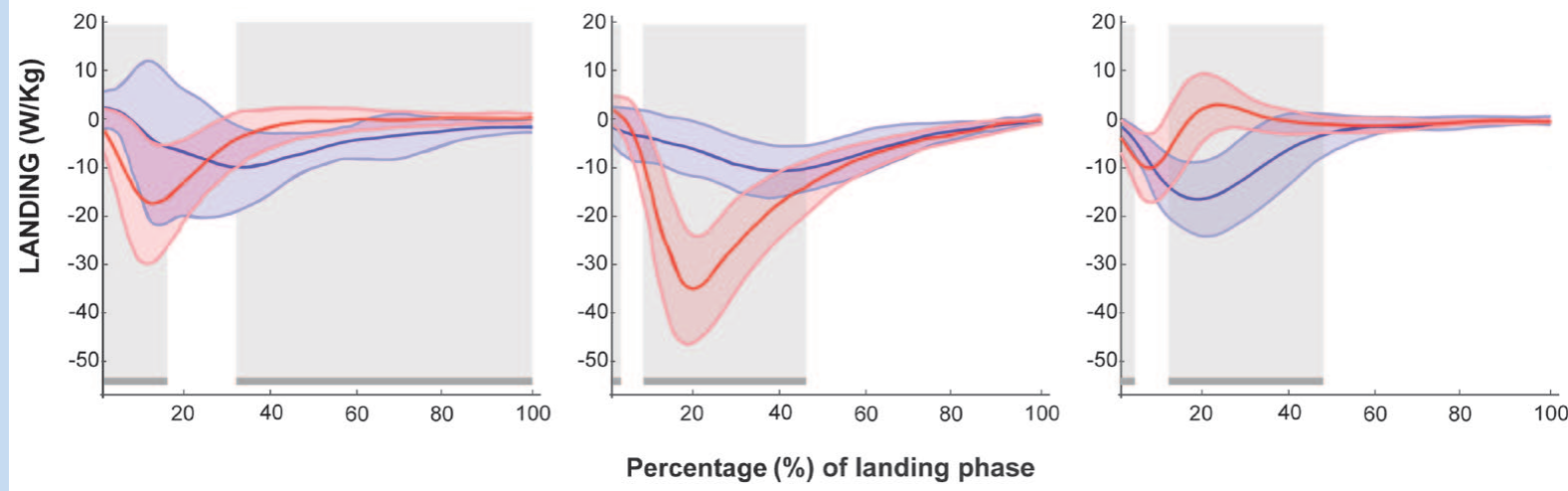

Vertical hop

Horizontal hop

Figure 2. Mean ( \pm SD cloud) of net joint power (W/kg) at the hip, knee, and ankle for the vertical (blue line) and horizontal (red line) hop, during propulsive and landing phases. Data were time-normalized as a percentage $(0 \%-100 \%)$ of each phase. Horizontal bars/gray shading indicate significant differences between the vertical and horizontal hop. For the propulsion phase, the peaks in activation occurred in a proximal to distal sequence (see upper panel) whereas for landing, there was no similar clear temporal sequence.

quadriceps peak force compared with horizontal hop distance $)^{21}$ is associated with $12.96 \%$ variance explained $\left(r^{2}\right)$. This matches the present data closely (12.9\% contribution of the knee to horizontal hop). Similarly, other researchers reported that aspects of knee strength can explain 13\% to $25 \%$ of the variance of horizontal hop performance. ${ }^{21,25,31}$ Conversely, stronger correlations have been reported between knee extension strength and vertical hop height ranging from 0.51 to $0.71,9,19,26$ which corresponds to $26 \%$ to $50 \%$ of the variance explained, respectively. These data appear in line with the results of the present study showing the knee joint accounting for approximately a third of the total work done.

\section{Spatiotemporal}

In both tasks, hip, knee, and ankle joints worked sequentially to perform these explosive movements. Peak power was observed earliest in the hip, then moved distally to the knee, and finally, the ankle during the propulsive phase. During landing, things were not so simple. After landing from the vertical hop, the ankle first absorbed with the hip and knee following roughly at the same time. When landing from the horizontal hop, the hip and ankle initiated the absorption for a short time but most of the work was done by the knee joint, which continued until the end of the landing phase (Figure 2).

\section{Implications for Clinical Testing}

During the horizontal hop, the knee joint contributed little to the propulsive phase, suggesting that knee joint function is poorly estimated by measuring single-leg hop distance. Previous research has shown off-loading of the anterior cruciate ligamentreconstructed leg despite symmetrical horizontal hop distance. ${ }^{42}$ A recent systematic review ${ }^{18}$ concluded that measuring only hop distance is insufficient to fully assess knee function after anterior cruciate ligament reconstruction, and this assumption seems plausible given the relative contribution of the knee joint in energy generation at the task. It has been suggested that patients may adopt compensation strategies to shift the work from the knee. Therefore single-leg hop performance is a measurement of 


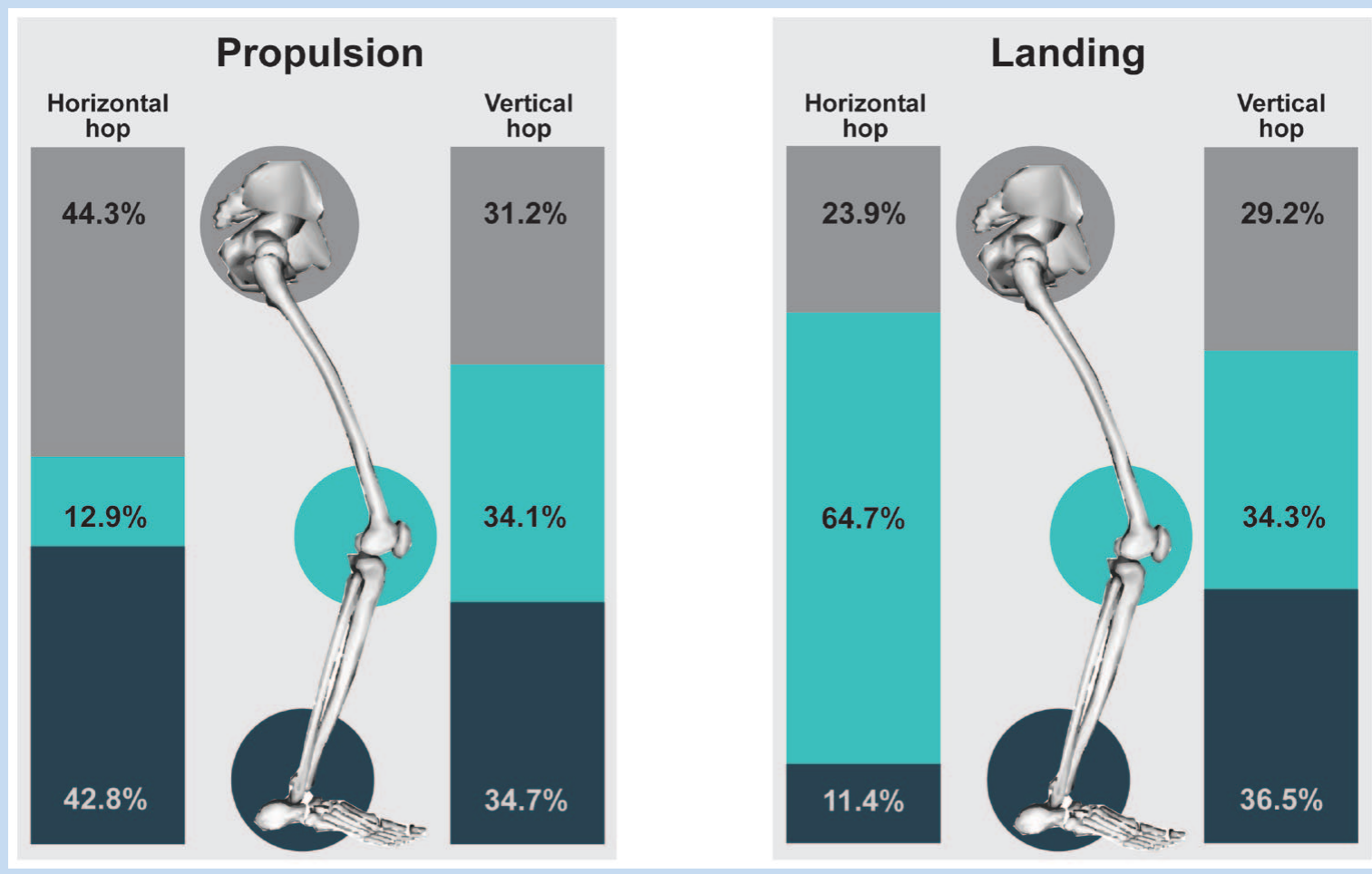

Figure 3. Percentage work contributions from the hip (gray), knee (turquoise), and ankle (teal) joints during horizontal and vertical hops, for the propulsive (left) and landing (right) phase. During the vertical hop, each joint contributes approximately $1 / 3$ of the total work, both in propulsive and landing phases. For the horizontal hop, the hip and ankle predominantly drive the movement with a total of $87 \%$ while knee joint contributes $13 \%$. Conversely, during landing the knee joint is the main work absorber, performing $65 \%$ of the total work.

Table 2. Differences in spatiotemporal, peak power, work, and joint contribution between vertical and horizontal hops ${ }^{a}$

\begin{tabular}{|l|l|c|c|c|c|c|c|c|c|}
\hline & & \multicolumn{9}{|c}{ Propulsion } & \multicolumn{3}{c|}{ Landing } \\
\hline & & Horizontal & Vertical & $\boldsymbol{P}$ & $\boldsymbol{d}$ & Horizontal & Vertical & $P$ & \multicolumn{1}{c|}{$\boldsymbol{d}$} \\
\hline Peak power, W/kg & Hip joint & $14.32 \pm 2.27$ & $11.36 \pm 2.84$ & $<0.001$ & 1.16 & $-27.67 \pm 9.62$ & $-22.28 \pm 13.85$ & 0.027 & 0.46 \\
\hline & Knee joint & $6.91 \pm 2.43$ & $12.11 \pm 2.48$ & $<0.001$ & 2.12 & $-43.16 \pm 11.19$ & $-17.01 \pm 3.84$ & $<0.001$ & 3.48 \\
\hline & Ankle joint & $24.85 \pm 4.24$ & $16.17 \pm 3.41$ & $<0.001$ & 2.27 & $-14.78 \pm 6.68$ & $-21.38 \pm 5.84$ & $<0.001$ & 1.05 \\
\hline Joint work, J/Kg & Hip joint & $2.10 \pm 0.42$ & $1.36 \pm 0.38$ & $<0.001$ & 1.85 & $-1.01 \pm 0.32$ & $-1.06 \pm 0.53$ & 0.594 & NA \\
\hline & Knee joint & $0.62 \pm 0.25$ & $1.49 \pm 0.39$ & $<0.001$ & 2.69 & $-2.78 \pm 0.66$ & $-1.22 \pm 0.46$ & $<0.001$ & 2.76 \\
\hline & Ankle joint & $2.04 \pm 0.40$ & $1.51 \pm 0.31$ & $<0.001$ & 1.52 & $-0.48 \pm 0.22$ & $-1.21 \pm 0.33$ & $<0.001$ & 2.69 \\
\hline & Total work & $4.77 \pm 0.78$ & $4.35 \pm 0.78$ & 0.007 & 0.53 & $-4.27 \pm 0.85$ & $-3.49 \pm 0.74$ & $<0.001$ & 0.97 \\
\hline & Hip joint & $44.3 \pm 6.6$ & $31.2 \pm 5.7$ & $<0.001$ & 2.13 & $23.9 \pm 6.8$ & $29.2 \pm 9.6$ & 0.001 & 0.65 \\
\hline & Knee joint & $12.9 \pm 4.5$ & $34.1 \pm 6.4$ & $<0.001$ & 3.89 & $64.7 \pm 5.8$ & $34.3 \pm 9.3$ & $<0.001$ & 4.04 \\
\hline & Ankle joint & $42.8 \pm 4.5$ & $34.7 \pm 4.9$ & $<0.001$ & 1.72 & $11.4 \pm 5.4$ & $36.5 \pm 12.1$ & $<0.001$ & 2.87 \\
\hline
\end{tabular}

NA, not applicable.

Values are presented as mean \pm SD. Values for propulsion phase are positive, and for landing they are negative. 
total lower extremity function and is not indicative of performance at a particular joint. ${ }^{24}$ Future research could examine relative work contributions of the different joints comparing injured and uninjured legs independent of hop distance and assist in clinical reasoning during rehabilitation.

During vertical hop, all 3 joints contributed roughly equally during the propulsive phase. Accordingly, dysfunction in any one of them may relatively equally affect hop performance (maximum height). Vertical hop height might therefore be more sensitive to knee asymmetry than the horizontal hop performance. The higher knee joint contribution in the vertical compared with horizontal can explain the delay in athletes achieving 90\% hop height symmetry after anterior cruciate ligament reconstruction. ${ }^{34}$

These 2 tasks are potential screening tools for the ability to generate or absorb power across lower limb joints.

Incorporation of multijoint power testing during the propulsive and landing phases of vertical and horizontal jumps might lead to identification of asymmetries in lower limb joints and to more targeted intervention during rehabilitation.

\section{Clinical Implications}

A systematic review identified the single-leg hop for distance tests as the only "clinician-friendly" tests that discriminated knee function after anterior cruciate ligament reconstruction. ${ }^{13}$ We suggest that the hop for distance performance measuredistance hopped-is mostly a reflection of the hip and ankle. Vertical hop has a higher performance contribution from the knee and may better reflect knee function than horizontal hop.

Identifying at a joint-level power absorption and generation during a functional task may be clinically useful in providing more specific information to guide the design of better targeted training. For example, the current data for healthy adults can be used as a comparison for an injured athlete and then used to direct training to a specific joint and mode of contraction. This may allow earlier identification and correction of inappropriate compensatory strategies in injured athletes' rehabilitation. Importantly, the current data are for male patients only. Knee ligament injury has a higher burden in female patients, and may be related to biomechanical differences in gait, hopping, or jumping. ${ }^{17,20}$

\section{Limitations}

These tests were performed on healthy recreational athletes in a controlled laboratory environment that is not indicative of the environment (surface, perturbations), intensity (recreational vs competitive athletes), or sport demands (complex multiplanar movements) during game situations. Importantly, it is not known if similar patterns exist after injury. Also, the recruitment of only male participants means these results are not applicable to their female counterparts. Inverse dynamics analyses have limitations and cannot account for the effects of co-contraction, biarticular muscles, or elastic energy storage and return.

\section{CONCLUSION}

There is little similarity in physical demands between vertical and horizontal jump. The current study shows performance on a vertical hop to be relatively equally apportioned to the hip, knee, and ankle joints. Conversely horizontal hop performance is primarily a function of the hip and ankle with a knee contribution limited to $13 \%$. We suggest that the horizontal hop test distance should be considered a poor discriminator of knee function and performance for these reasons. Given the different biomechanical demands of each functional task, both can be used in lower limb evaluation, but their differences need to be understood.

\section{REFERENCES}

1. Barber SD, Noyes FR, Mangine RE, McCloskey JW, Hartman W. Quantitative assessment of functional limitations in normal and anterior cruciate ligamentdeficient knees. Clin Orthop. 1990;255:204-214.

2. Barfod KW, Feller JA, Hartwig T, Devitt BM, Webster KE. Knee extensor strength and hop test performance following anterior cruciate ligament reconstruction. Knee. 2019;26:149-154

3. Bezodis NE, Salo AI, Trewartha G. Excessive fluctuations in knee joint moments during early stance in sprinting are caused by digital filtering procedures. Gait Posture. 2013;38:653-657.

4. Bolgla LA, Keskula DR. Reliability of lower extremity functional performance tests. J Orthop Sports Phys Ther. 1997;26:138-142.

5. Brosky JA Jr, Nitz AJ, Malone TR, Caborn DN, Rayens MK. Intrarater reliability of selected clinical outcome measures following anterior cruciate ligament reconstruction. J Orthop Sports Phys Ther. 1999;29:39-48.

6. Cohen J. Statistical Power Analysis for the Behavioral Sciences. 2nd ed. Lawrence Erlbaum; 1988.

7. Considine WJ, Sullivan WJ. Relationship of selected tests of leg strength and leg power on college men. Res Q Exerc Sport. 1973;44:404-416.

8. Davis RB, Õunpuu S, Tyburski D, Gage JR. A gait analysis data collection and reduction technique. Hum Mov Sci. 1991;10:575-587.

9. Fischer F, Blank C, Dünnwald T, et al. Isokinetic extension strength is associated with single-leg vertical jump height. Orthop J Sports Med. 2017;5:2325967117736766.

10. Fitzgerald GK, Lephart SM, Hwang JH, Wainner RS. Hop tests as predictors of dynamic knee stability. J Orthop Sports Phys Ther. 2001;31:588-597.

11. Fukashiro S, Komi PV. Joint moment and mechanical power flow of the lower limb during vertical jump. Int J Sports Med. 1987;8(suppl 1):15-21.

12. Hamilton RT, Shultz SJ, Schmitz RJ, Perrin DH. Triple-hop distance as a valid predictor of lower limb strength and power. J Athl Train. 2008;43:144-151.

13. Hegedus EJ, McDonough S, Bleakley C, Cook CE, Baxter GD. Clinician-friendly lower extremity physical performance measures in athletes: a systematic review of measurement properties and correlation with injury, part 1 . The tests for knee function including the hop tests. Br J Sports Med. 2015;49:642-648.

14. Hubley CL, Wells RP. A work-energy approach to determine individual joint contributions to vertical jump performance. Eur J Appl Physiol Occup Physiol. $1983 ; 50: 247-254$

15. Järvelä T, Kannus P, Latvala K, Järvinen M. Simple measurements in assessing muscle performance after an ACL reconstruction. Int J Sports Med. 2002;23:196-201.

16. Kale M, Asçi A, Bayrak C, Açikada C. Relationships among jumping performances and sprint parameters during maximum speed phase in sprinters. J Strength Cond Res. 2009;23:2272-2279.

17. Koga H, Nakamae A, Shima Y, et al. Mechanisms for noncontact anterior cruciate ligament injuries: knee joint kinematics in 10 injury situations from female team handball and basketball. Am J Sports Med. 2010;38:2218-2225.

18. Kotsifaki A, Korakakis V, Whiteley R, Van Rossom S, Jonkers I. Measuring only hop distance during single leg hop testing is insufficient to detect deficits in knee function after ACL reconstruction: a systematic review and meta-analysis. Br J Sports Med. 2020;54:139-153. 
19. Laudner K, Evans D, Wong R, et al. Relationship between isokinetic knee strength and jump characteristics following anterior cruciate ligament reconstruction. Int J Sports Phys Ther. 2015;10:272-280.

20. Leppanen M, Pasanen K, Kujala UM, et al. Stiff landings are associated with increased ACL injury risk in young female basketball and floorball players. Am J Sports Med. 2017; 45:386-393.

21. Nagai T, Schilaty ND, Laskowski ER, Hewett TE. Hop tests can result in higher limb symmetry index values than isokinetic strength and leg press tests in patients following ACL reconstruction. Knee Surg Sports Traumatol Arthrosc. 2020;28:816-822.

22. Negrete R, Brophy J. The relationship between isokinetic open and closed chain lower extremity strength and functional performance. J Sport Rehabil. 2000;9:46-61.

23. Noyes FR, Barber SD, Mangine RE. Abnormal lower limb symmetry determined by function hop tests after anterior cruciate ligament rupture. Am J Sports Med. 1991;19:513-518.

24. Orishimo KF, Kremenic IJ, Mullaney MJ, McHugh MP, Nicholas SJ. Adaptations in single-leg hop biomechanics following anterior cruciate ligament reconstruction. Knee Surg Sports Traumatol Arthrosc. 2010;18:1587-1593.

25. Palmieri-Smith RM, Lepley LK. Quadriceps strength asymmetry after anterior cruciate ligament reconstruction alters knee joint biomechanics and functional performance at time of return to activity. Am J Sports Med. 2015;43:1662-1669.

26. Petschnig R, Baron R, Albrecht M. The relationship between isokinetic quadriceps strength test and hop tests for distance and one-legged vertical jump test following anterior cruciate ligament reconstruction. J Orthop Sports Phys Ther. 1998;28:23-31.

27. Reid A, Birmingham TB, Stratford PW, Alcock GK, Giffin JR. Hop testing provides a reliable and valid outcome measure during rehabilitation after anterior cruciate ligament reconstruction. Phys Ther. 2007;87:337-349.

28. Ridderikhoff A, Batelaan JH, Bobbert MF. Jumping for distance: control of the external force in squat jumps. Med Sci Sports Exerc. 1999;31:1196-1204.

29. Robertson D, Fleming D. Kinetics of standing broad and vertical jumping. Can J Sport Sci. 1987;12:19-23.

30. Schache AG, Brown NA, Pandy MG. Modulation of work and power by the human lower-limb joints with increasing steady-state locomotion speed. J Exp Biol. 2015;218(pt 15):2472-2481.
31. Schmitt LC, Paterno MV, Hewett TE. The impact of quadriceps femoris strength asymmetry on functional performance at return to sport following anterior cruciate ligament reconstruction. J Orthop Sports Phys Ther. 2012;42:750-759

32. Swearingen J, Lawrence E, Stevens J, Jackson C, Waggy C, Davis DS. Correlation of single leg vertical jump, single leg hop for distance, and single leg hop for time. Phys Ther Sport. 2011;12:194-198.

33. Taylor JB, Ford KR, Nguyen AD, Shultz SJ. Biomechanical comparison of singleand double-leg jump landings in the sagittal and frontal plane. Orthop J Sports Med. 2016;4:2325967116655158.

34. Thomeé R, Neeter C, Gustavsson A, et al. Variability in leg muscle power and hop performance after anterior cruciate ligament reconstruction. Knee Surg Sports Traumatol Arthrosc. 2012;20:1143-1151.

35. Undheim MB, Cosgrave C, King E, et al. Isokinetic muscle strength and readiness to return to sport following anterior cruciate ligament reconstruction: is there an association? A systematic review and a protocol recommendation. Br J Sports Med. 2015;49:1305-1310.

36. van Melick N, Meddeler BM, Hoogeboom TJ, Nijhuis-van der Sanden MWG, van Cingel REH. How to determine leg dominance: the agreement between self-reported and observed performance in healthy adults. PLoS One 2017;12:e0189876.

37. Vanezis A, Lees A. A biomechanical analysis of good and poor performers of the vertical jump. Ergonomics. 2005;48:1594-1603.

38. Viitasalo J. Anthropometric and physical performance characteristics of male volleyball players. Can J Appl Sport Sci. 1982;7:182-188.

39. von Elm E, Altman DG, Egger M, et al. The Strengthening the Reporting of Observational Studies in Epidemiology (STROBE) statement: guidelines for reporting observational studies. J Clin Epidemiol. 2008;61:344-349.

40. Wilk KE, Romaniello WT, Soscia SM, Arrigo CA, Andrews JR. The relationship between subjective knee scores, isokinetic testing, and functional testing in the ACL-reconstructed knee. J Orthop Sports Phys Ther. 1994;20:60-73.

41. Winter DA. Biomechanics and Motor Control of Human Movement. Wiley; 2009.

42. Wren TAL, Mueske NM, Brophy CH, et al. Hop distance symmetry does not indicate normal landing biomechanics in adolescent athletes with recent anterior cruciate ligament reconstruction. J Orthop Sports Phys Ther. 2018;48:622-629.

For article reuse guidelines, please visit SAGE's website at http://www.sagepub.com/journals-permissions 